A0017: Repeated stages of fugue in patients with epilepsy: case series

Yadav J.S., ${ }^{1}$ Srivstava A.S., ${ }^{1}$ Yadav Jyoti, ${ }^{1}$ Chaurasia R.N. ${ }^{1}$ ${ }^{1}$ Institute of Medical Sciences, Banaras Hindu University, Varanasi, Uttar Pradesh, India

Objective: To find out prolonged recurrent states of fugue in patients with epilepsy.

Methodology We have included five cases from psychiatry and child guidance OPD, Institute of Medical Sciences, B.H.U., Varanasi. The initial evaluation was done by consultant in-charge psychiatry and CGC OPD. The investigations were sent and finally all patients were referred to neurology OPD and the neurologist made final diagnosis. For further confirmation, CT scan/MRI head and EEG recording of all patient were done.

Results: The age of all five cases varied from 12 to 45 years and out of five, two were females; they belonged to lower socioeconomic status and were educated. Two patients had positive family history of epilepsy but their siblings did not have similar complaints. All five patients have initial agitation followed by wandering aimlessly for varied durations. Findings of CT scan, laboratory investigations were normal but EEG in all five patients was abnormal.

Conclusion: The fugue stages in psychological disorder differ from patients with epilepsy/organic causes of fugue, in dissociative fugue, there are temporal relation found with psychological trauma. In those phases, patient maintains his safety with different identity and the initial symptom of abnormal behavior unlikely occurs. We found in these cases that patients were not able to maintain themselves during fugue stage and their initial presentations were agitation and the important differences were found in EEG reports, those were abnormal.

\section{A0018: Hemispherotomy in Adults. Is It Safe?-A Prospective Observational Study in Comparison to Children}

ఏitin Bajaj, ${ }^{1}$ P. Sarat Chandra, ${ }^{1}$ Bhargavi Ramanujam, ${ }^{2}$ Shabari Girishan, ${ }^{1}$ Ramesh Doddamani, ${ }^{1}$ Manjari Tripathi ${ }^{2}$

${ }^{1}$ Department of Neurosurgery, All India Institute of Medical Sciences, New Delhi, India

2Department of Neurology, All India Institute of Medical Sciences, New Delhi, India

Introduction: Hemispherotomy (HS) is a safe and effective procedure for drug-resistant epilepsy in appropriately selected patients. There are few publications for HS in adults with less than 100 patients reported till date. This series compares the HS in adults and pediatric age groups, which is first of its kind.

Methods: Data were prospectively collected for HS patients during April 2014 to August 2017. Cutoff age was 18 years. Comparison between the groups was done for seizure onset, duration of epilepsy, frequency of seizures, number of drugs, intraoperative blood loss, postoperative seizure control postoperative stay, postoperative motor functions, and preoperative and postoperative intelligence quotient.
Results: Total 55 pediatric and nine adults underwent HS. The seizure onset was earlier in children and duration of epilepsy was longer in adults. The frequency of seizures per day was $14.62 \pm 2.57$ in children, while in adults was $7.71 \pm$ 5.21 per day, with the p-value of 0.49 . Mean number of drugs was similar in the preoperative and postoperative periods in both. In this study, $85.5 \%$ of children and $88.9 \%$ of adults had class I seizure outcome $(p=-0.59)$. Blood loss and postoperative stay were similar in both the groups. No patient had permanent motor deficit. There was transient worsening of power in one pediatric patient and in four adult patients. IQ remained same in both the groups. One adult patient had meningitis and another had hydrocephalous requiring shunt placement.

Conclusion: Hemispherotomy is as safe and effective procedure in adults as in children in appropriately selected patients.

A0019: Benzimidazole Group of Drugs Possess Significant Anticonvulsant Properties in Experimentally Induced Animal Models

Chavan M.D., ${ }^{1}$ Karamthoti M.B., ${ }^{2}$ Kurra S.B. ${ }^{1}$

'Department of Pharmacology, MIMS, Tamil Nadu, India

2Department of Physiology, MIMS, Tamil Nadu, India

Objective: To study the anticonvulsant properties of benzimidazole group of drugs in experimentally induced animal models.

Methods: In this study, experimental animals were induced convulsions by two different Methods and which included, electrically induced with the help of electroconvulsiometer with the placement of electrodes over the ear and the subcutaneous (SC) administration of pentylenetetrazole ( $p$ TZ) in the dose of $70 \mathrm{mg} / \mathrm{kg} \mathrm{BW}$.

The study included a total of eight different groups with group I: control for PTZ, group II: standard for PTZ (sodium valproate), group III: benzimidazole group drug 1 for PTZ, group IV: benzimidazole group drug-2 for PTZ, group V: control for MES, group VI: standard for MES (diphenylhydantoin), group VII: benzimidazole group drug-1 for MES, and group VIII: benzimidazole group drug-2 for MES. Statistical analysis was carried with the help of statistical software (GraphPad Instat version 3.06), 32 bit for Windows and Results were expressed as mean/median \pm SE.

Results: The Results of the study indicated statistically significant rise in onset of seizures (in seconds) in groups III and IV when compared with the control (group I) in chemically induced convulsion model with p-value $<0.05$. It was also noted that the duration of seizures significantly declined in both the experimental test drug groups (i.e., groups III and IV) when compared with the control group (group I) with p-value less than 0.05 . Similarly, the total number of seizures in 1 hour also reduced significantly in both the experimental test drug groups (i.e., benzimidazole group drugs 1 and 2 ) in comparison to the group I $(p<0.05)$. The study Results confirmed that, for all the above four parameters, there was no statistically significant difference seen among the standard and experimental test drug groups with p-value of more than 0.05. In MES 